subgroup of patients with lower initial body mass index $\left(<17.5 \mathrm{~kg} / \mathrm{m}^{2}\right.$ at the beginning of treatment) there was some suggestion that patients receiving MANTRA showed greater weight gain than those receiving SSCM, but this was not statistically significant $(P=0.15)$ as the study was not powered to detect subgroup differences. Second, the original New Zealand trial - where SSCM compared well against cognitive-behavioural treatment and interpersonal therapy - included many patients who had a relatively mild, less chronic form of anorexia. In this earlier trial, SSCM effects seemed to wane in the long term. ${ }^{1}$

Second, contrary to Gutierrez \& Carrera's assertion, there is plenty of evidence that the personality features, neuropsychological profile (thinking style) and aspects of altered socioemotional processing found in anorexia are not just an epiphenomenon of malnutrition but have trait characteristics which are accentuated in the starved state. ${ }^{2}$

Taken together these findings suggest a definite place for SSCM, especially in the treatment of less severe cases of anorexia. It may be that a more complex treatment such as MANTRA, which is trait-focused and where patients are taught skills that help them to tackle a range of maintaining factors, is more effective in more severe cases. Our trial was too small to tease this out. However, a larger study is now under way that should be able to answer this question. ${ }^{2}$

To suggest an 'either/or' dichotomy between a treatment focus on self or starvation seems remarkably simplistic to us. In fact, if an exclusive focus on reducing starvation was the key curative step in treatment, in-patient refeeding for anorexia should be used much more often, as this reverses poor nutrition most quickly. Yet, in-patient treatment has significant problems: it is unacceptable to many patients and has high relapse rates.

In a large-scale international survey of patients with eating disorders and their families, there was strong agreement between these stakeholders that specialist expertise and personal qualities of staff, expert psychological interventions and nutritional assistance (advice and intervention) combined are the key components of effective treatments and services. ${ }^{3}$

Clearly, we are a long way away from having a cure for adults with anorexia. Given the very limited evidence base, there is still much to learn about what works for whom and at which stage of illness. The past few years have seen the burgeoning of neuroscience data related to anorexia nervosa, which opens the way to treatments targeted at dysfunctional neurocircuitry. ${ }^{4,5}$ Ultimately, we predict that significant improvements in treatment outcomes in adults with anorexia are only going to be achieved through adding such 'targeted brain-directed' adjuncts to talking therapies and nutritional intervention.

1 Carter FA, Jordan J, McIntosh VV, Luty SE, McKenzie JM, Frampton CM, et al. The long-term efficacy of three psychotherapies for anorexia nervosa: a randomized, controlled trial. Int J Eat Disord 2011; 44: 647-54.

2 Treasure J, Schmidt U. The Cognitive-Interpersonal Maintenance Model of Anorexia Nervosa revisited: a summary of the evidence for cognitive, socio-emotional and interpersonal predisposing and perpetuating factors. Int J Eat Disord 2013; in press.

3 Nishizono-Maher A, Escobar-Koch T, Ringwood S, Banker J, van Furth E, Schmidt $U$. What are the top five essential features of a high quality eating disorder service? A comparison of the views of US and UK eating disorder sufferers, carers and health professionals. Eur Eat Disord Rev 2010; 20 Dec. (Epub ahead of print.)

4 Van den Eynde F, Guillaume S, Broadbent $\mathrm{H}$, Campbell IC, Schmidt U. Repetitive transcranial magnetic stimulation in anorexia nervosa: a pilot study. Eur Psychiatry 2013: 28: 98-101.

5 Lipsman N, Woodside DB, Giacobbe P, Hamani C, Carter JC, Norwood SJ, et al. Subcallosal cingulate deep brain stimulation for treatment-refractory anorexia nervosa: a phase 1 pilot trial. Lancet 2013; doi: 10.1016/S01406736(12)62188-6. (Epub ahead of print.)
Ulrike Schmidt, Section of Eating Disorders, Institute of Psychiatry, De Crespigny Park, London SE5 8AF, UK. Email: ulrike.schmidt@kcl.ac.uk; Anna Oldershaw, Lot Sternheim, Kate Tchanturia, Janet Treasure, Section of Eating Disorders, Fatima Jichi, Sabine Landau, Department of Biostatistics, King's College London, Institute of Psychiatry, UK, Helen Startup, Geoffrey Wolff, Michael Rooney, Eating Disorders Uny, Unit, South London and Maudsley NHS Foundation Trust, UK; Virginia McIntosh, Jennifer Jordan, University of Otago, Department of Psychological Medicine, Christchurch, New Zealand

doi: $10.1192 / \mathrm{bjp} .202 .5 .384 a$

\section{Spirituality is not bad for our mental health}

We note with interest the conclusion of King et al's study, ${ }^{1}$ which states that 'people who have a spiritual understanding of life in the absence of a religious framework are vulnerable to mental disorder'. A second, equally important finding is that 'religious people were similar to those who were neither religious nor spiritual with regard to the prevalence of mental disorders, except that the former were less likely to have ever used drugs [...] or be a hazardous drinker'. This lack of difference, as with the key conclusion concerning those who are spiritual but not religious, runs counter to the substantial body of evidence collated by Koenig et $a l,{ }^{2-4}$ who conclude that religion/spirituality are generally associated with better mental health.

King et al point out that 'the cross-sectional nature of the data means that we cannot attribute cause and effect to any relationship between spiritual beliefs and mental health', and they draw attention to important differences between the UK and North America (where the bulk of previous research has been conducted). The headline conclusion of the study may nonetheless leave professionals and others with the impression that 'spirituality' is bad for one's health, an impression that we believe would be mistaken.

Our post-modern culture is geared increasingly to a way of life that does not question deeply such things as the meaning of birth and death, why we are here and what it is all for. Instead, social norms often emphasise aspiration to goals of material ambition and success. For many, it seems that this can result in estrangement from the most fundamental spiritual needs and values of humankind (a theme that comes up at meetings of the Royal College of Psychiatrists' Spirituality and Psychiatry Special Interest Group).

With the decline in religious observance, the numbers of 'spiritual but not religious' (19\% in this study) are rising, and perhaps more so in the UK than in the USA. Wrestling with the deepest questions about life is in the nature of the human condition. However, without a religious faith that can also provide a person with both community and support, the road is long and hard and the journey often a lonely one. Previous research (Pargament, ${ }^{5}$ pp. 111-128) suggests that spiritual struggles have the potential for either good or bad mental health outcomes, and we wonder whether the kind of society in which we are now living is less than supportive of the good outcome.

We know that spiritually informed therapies are effective in the field of substance misuse, ${ }^{6}$ and mindfulness-based approaches derived from spiritual practice are now recommended by the National Institute for Health and Clinical Excellence for relapse prevention of depression. ${ }^{7}$ Further, we believe that spirituality has an important secular dimension which is finding expression in the recovery movement in psychiatry.

We must therefore guard against any misreading of this study by King et al that would suggest spirituality is bad for mental health. We do, however, support strongly research that is able both to delineate causal pathways and provide comparison between the cultures and contexts of the USA and the UK. 


\section{Declaration of interest}

A.P. and C.C.H.C. are both members of the Executive Committee of the Royal College of Psychiatrists' Spirituality and Psychiatry Special Interest Group. However, the views expressed in this letter are their own and not necessarily those held by the Group as a whole. C.C.H.C. is an Anglican Priest and Director of the Project for Spirituality, Theology \& Health at Durham University.

1 King M, Marston L, McManus S, Brugha T, Meltzer H, Bebbington P. Religion spirituality and mental health: results from a national study of English households. Br J Psychiatry 2013; 202: 68-73.

2 Koenig HG, Mccullough ME, Larson DB. Handbook of Religion and Health. Oxford University Press, 2001.

3 Koenig HG, King DE, Carson VB. Handbook of Religion and Health (2nd edn) Oxford University Press, 2012.

4 Koenig HG. Religion, spirituality and health: the research and clinical implications. ISRN Psychiatry 2012; doi: 10.5402/2012/278730.

5 Pargament KI. Spiritually Integrated Psychotherapy: Understanding and Addressing the Sacred. Guilford Press, 2011.

6 Cook CCH. Substance misuse. In Spirituality and Psychiatry (eds C Cook A Powell, A Sims): 139-68. RCPsych Publications, 2009.

7 National Institute for Health and Clinical Excellence. Depression: The Treatment and Management of Depression in Adults (Clinical Guideline CG90). NICE, 2009.

Christopher C. H. Cook, Professor, Department of Theology \& Religion, Durham University, UK. Email: c.c.h.cook@durham.ac.uk; Andrew Powell, formerly: Consultant Psychotherapist and Senior Lecturer in Psychiatry, St George's Hospital and University of London; and Consultant Psychotherapist and Honorary Senior Lecturer, Warneford Hospital and University of Oxford, UK

doi: 10.1192/bjp.202.5.385

Author's reply: Cook \& Powell are surprised that our findings run counter to research conducted in the USA. They are also concerned that people may conclude from our data that spirituality is bad for mental health. Rather than bad, our main finding is that a religious or spiritual life view confers no advantage in terms of mental health. Our results are not so unusual. Although reviews have suggested that religious and spiritual beliefs and practices are associated with better mental health, the evidence often comes from poor-quality studies and effect sizes reported are small. This is not surprising from a theological point of view; the idea that religious people are protected from the impact of life's difficulties runs counter to the theology of most major world religions. ${ }^{1}$ Furthermore, the evidence base that spiritually informed therapies are effective is tiny, partly because funding for trials is hard to obtain and there have been very few well-designed studies. Religious belief and practice has its main impact on health through lifestyle habits (e.g. less consumption of tobacco and alcohol) and social support. Also, as Cook \& Powell note, the context in Europe is quite different to that in the USA. Professing a religious or spiritual belief in Europe may be regarded as strange or even derisory. Such beliefs and practice are more mainstream in the Americas, although even there the occurrence of such beliefs is declining.

In findings from a large prospective study across Europe, published after this paper, we have shown again that holding a spiritual or religious life view may be associated with later mental health problems, but that the effects are weak. More importantly, however, we confirmed that there was no mental health advantage for such beliefs. ${ }^{2}$ These prospective data give clues to the direction of the association. It seems that holding a spiritual life view predisposes people to depression. As Cook \& Powell say, a spiritual search may often be a 'lonely one'. However, it remains possible that the search for spiritual answers does not itself cause depression; rather, people already vulnerable to depression search for spiritual answers.

1 Schumann JJ, Meador KG. Heal Thyself: Spirituality, Medicine, and the Distortion of Christianity. Oxford University Press, 2003.

2 Leurent B, Nazareth I, Bellón-Saameño J, Geerlings MI, Maaroos H, Saldivia S, et al. Spiritual and religious beliefs as risk factors for the onset of major depression: an international cohort study. Psychol Med 2013; Jan 29: 1-12. doi: 10.1017/S0033291712003066. (Epub ahead of print.)

Michael King, Unit of Mental Health Sciences, Faculty of Brain Sciences, University College London Medical School, Charles Bell House, 67-73 Riding House Street, London W1W 7EH, UK. Email: michael.king@ucl.ac.uk

doi: $10.1192 /$ bjp.202.5.386 